\title{
ESTUDO DO PROCESSO DE ADSORÇÃO DE AZUL DE METILENO EM MEIO AQUOSO EMPREGANDO SILICA SBA-15 COMO ADSORVENTE
}

\author{
G. R. F. GONÇALVES ${ }^{1 *}$, O. R. R GANDOLFI ${ }^{1}$, J. G. PIMENTEL ${ }^{2}$, C. M. VELOSO ${ }^{3}$, \\ R. C. I. FONTAN ${ }^{4}$, R. C. F. BONOMO ${ }^{4}$
}

${ }^{1}$ Universidade Estadual do Sudoeste da Bahia, Mestrando em Engenharia e Ciência de Alimentos

${ }^{2}$ Universidade Estadual do Sudoeste da Bahia, Mestrando em Ciências Ambientais

${ }^{3}$ Universidade Estadual do Sudoeste da Bahia, Departamento de Ciências Naturais

${ }^{4}$ Universidade Estadual do Sudoeste da Bahia, Departamento de Tecnologia Rural e Animal *e-mail: gabriel_ramosfg@hotmail.com

\begin{abstract}
RESUMO
A sílica SBA-15 é um material mesoporoso com grande potencial de aplicação, inclusive no processo de adsorção. Levando esse fator em consideração objetivou-se nesse trabalho sintetizar e caracterizar a sílica SBA- 15 nas temperaturas de $80^{\circ} \mathrm{C}, 105^{\circ} \mathrm{C}$ e $120^{\circ} \mathrm{C}$ e estudar a sua capacidade de adsorção do corante azul de metileno em meio aquoso em diferentes condições de concentração e $\mathrm{pH}$. Foi verificado que a sílica sintetizada na temperatura de $80^{\circ} \mathrm{C}$ apresentou maior eficiência de adsorção. Tal fato pode ser explicado pela maior área superficial dessa sílica, apresentando assim mais sítios de ligação, favorecendo a adsorção. A combinação que obteve uma maior adsorção no teste inicial foi a sílica sintetizada a $80^{\circ} \mathrm{C}$ com o azul de metileno na concentração de $100 \mathrm{mg} . \mathrm{L}^{-1}$. Posteriormente, foi verificado que o $\mathrm{pH} \mathrm{9,0} \mathrm{foi} \mathrm{o} \mathrm{que}$ obteve a maior eficiência de adsorção.
\end{abstract}

\section{INTRODUÇÃO}

A adsorção é um fenômeno físicoquímico em que o componente em fase líquida é transferido para a superfície de uma fase sólida. Os componentes que se unem à superfície são chamados adsorvatos, enquanto que a fase sólida que retém o adsorvato é chamada adsorvente. A remoção das moléculas a partir da superfície é chamada dessorção (MASEL, 1996). Diversos materiais podem ser utilizados como adsorventes: carvões ativados, sílicas mesoporosas, resinas trocadoras de ions, entre outros.

As sílicas mesoporosas são partículas de óxido de silício $\left(\mathrm{SiO}_{2}\right)$ com estrutura altamente organizada, que devido as suas propriedades físico-químicas apresentam potencial de aplicação em diferentes áreas, despertando grande interesse na comunidade científica. Esses materiais são capazes de interagir com átomos, íons e moléculas, não apenas em sua superfície, como também em seu interior.

Dentre as sílicas mesoporosas, a SBA15 vem sendo bastante estudada, com diversas possibilidades de aplicação. Esse material apresenta estrutura hexagonal com poros altamente ordenados e interligados, paredes relativamente espessas e alta estabilidade térmica, hidrotérmica e mecânica. A SBA-15 é sintetizada em meio ácido, utilizando como agente direcionador de estrutura um copolímero tribloco, o poli(óxido de etileno)poli(óxido de propileno)-poli(óxido de 
etileno), podendo agregar-se com espécies de silicatos, formando a sílica mesoporosa.Uma característica relevante da SBA-15 é a presença de uma grande quantidade de grupos silanóis livres $(\equiv \mathrm{Si}-\mathrm{OH})$, nas paredes dos poros, além de possuir propriedades estruturais e texturais, que aliadas a possibilidade de funcionalização de sua superfície, torna-se um material eficiente e seletivo para ser utilizado como adsorvente(YANG et al., 1997; ZHAO et al.,1998).

Diante do exposto, objetivou-se nesse trabalho sintetizar e caracterizar a sílica SBA15 nas temperaturas de $80^{\circ} \mathrm{C}, 105^{\circ} \mathrm{C}$ e $120^{\circ} \mathrm{C}$ e estudar a sua capacidade de adsorção do corante azul de metileno em meio aquoso em diferentes condições de concentração e pH.

\section{MATERIAIS E MÉTODOS}

O trabalho experimental foi realizado no Laboratório de Engenharia de Processos da Universidade Estadual do Sudoeste da Bahia, campus de Itapetinga.

\subsection{Síntese da Sílica SBA-15}

A síntese da sílica mesoporosa SBA-15 consistiu na adição da fonte de silício, tetraetilortosilicato (TEOS), a uma solução aquosa de ácido clorídrico, contendo um surfactante adequado (Pluronic P123). A mistura resultante permaneceu durante $20 \mathrm{~h}$ sob agitação à temperatura constante de $40^{\circ} \mathrm{C}$ na BOD. Em seguida a mesma foi transferida para autoclaves e levadas a estufa por $48 \mathrm{~h}$ para o envelhecimento na temperatura a ser sintetizada $\left(80{ }^{\circ} \mathrm{C}, 105{ }^{\circ} \mathrm{C}\right.$ ou $\left.120^{\circ} \mathrm{C}\right)$. O produto obtido foi separado por centrifugação, lavado com água destilada, filtrado e seco em estufa por $24 \mathrm{~h}$ a $60{ }^{\circ} \mathrm{C}$, removendo o surfactante por calcinação a $550^{\circ} \mathrm{C}$ por $6 \mathrm{~h}$ usando uma taxa de aquecimento de $2^{\circ} \mathrm{C} \cdot \mathrm{min}^{-1}$.

\subsection{Caracterização da Sílica}

\subsubsection{Difração de Raio X}

Utilizou-se a difração de Raios X para obter informações sobre $\mathrm{o}$ ordenamento estrutural das sílicas SBA-15. Os experimentos de difração de Raios X (DRX) foram realizados em um equipamento BRUKER modelo D8 ADVANCE utilizando radiação $\mathrm{CuK} \alpha$ (40 kW e $40 \mathrm{~mA}$ ) na faixa de $0,6^{\circ} \leq 2 \theta \leq 10^{\circ}$ e $10^{\circ} \leq 2 \theta \leq 80^{\circ}$. Utilizou-se a potência de $1600 \mathrm{~V}$, fenda de $0,2 \mathrm{~mm}$ e o método de escaneamento contínuo nas análises a ângulos acima de $10^{\circ}$. Nas análises a ângulos baixos $\left(0,6^{\circ} \leq 2 \theta \leq 10^{\circ}\right)$, utilizou-se a fenda $0,1 \mathrm{~mm}$, tamanho de passo para $2 \theta$ de $0,01^{\circ}$ com tempo de contagem de $1 \mathrm{~s}$ por passo.

2.2.2 Medida de porosidade e área superficial específica

As isotermas de adsorção e dessorção das amostras obtidas foram determinadas em um equipamento Micromeritcs modelo ASAP 2420, utilizando aproximadamente $0,15 \mathrm{~g}$ de amostra. Antes das medidas, as amostras foram submetidas a uma etapa de prétratamento. Posteriormente foram obtidas isotermas de adsorção e dessorção de nitrogênio a $-195,85{ }^{\circ} \mathrm{C}$. A área superficial específica foi determinada pela equação de BET (BRUNAUER, 1938). A distribuição de poros foi obtida a partir da isoterma de dessorção utilizando o método BJH (BARRET, 1951), enquanto que o volume dos microporos foi determinado pela análise t-plot a partir da isoterma de adsorção (LIPPENS, 1964).

\subsection{Obtenção das Condições Ótimas de Adsorção}

Inicialmente as amostras de sílica SBA15 preparadas nas 3 temperaturas de 
calcinação $\left(80^{\circ} \mathrm{C}, 105^{\circ} \mathrm{C}\right.$ e $\left.120^{\circ} \mathrm{C}\right)$ foram utilizadas para adsorver o azul de metileno nas concentrações de 100 ppm, 200 ppm e 300 ppm. Nesta etapa, utilizou-se aproximadamente $0,01 \mathrm{~g}$ da sílica nas concentrações testadas, onde foram acondicionadas em tubos contendo $5 \mathrm{ml} \mathrm{da}$ solução do corante em $\mathrm{pH}$ neutro. Os tubos foram mantidos sob agitação constante (20 rpm), em um aparato experimental, em temperatura ambiente por um período de $24 \mathrm{~h}$. Em seguida a solução foi centrifugada em centrífuga modelo MPW-350, e quantificou o corante remanescente por leitura direta em espectrofotômetro (Biochrom Libra S70), no comprimento de onda $665 \mathrm{~nm}$, que é o ponto onde ocorre o pico de absorbância do azul de metileno. Após essa etapa a eficiência de adsorção em todas as condições estudadas foram obtidas pela Equação 1 .

efic $=\left(\frac{V C_{i n}-V C}{V C_{i n}}\right) \times 100$

Onde: efic é a eficiência da adsorção (\%),V é o volume da solução $(\mathrm{mL}), \mathrm{C}_{\mathrm{in}}$ é a concentração inicial da solução $\left(m g . L^{-1}\right)$ e C é a concentração da solução no equilíbrio (mg. $\left.\mathrm{L}^{-1}\right)$.

Em seguida, foi realizado o estudo de $\mathrm{pH}$ que consistiu em acondicionar aproximadamente $0,01 \mathrm{~g}$ de sílica SBA-15 sintetizada na temperatura de $80^{\circ} \mathrm{C}$ em tubos contendo $5 \mathrm{~mL}$ de azul de metileno na concentração de $100 \mathrm{mg} . \mathrm{L}^{-1}$ e o ajuste de $\mathrm{pH}$ foi realizado utilizando solução tampão de fosfato de sódio para os valores de $\mathrm{pH}$ 3,0, 6,5 e 9,0, onde os tubos foram mantidos sob agitação constante $(20 \mathrm{rpm})$, em um aparato experimental, em temperatura ambiente por um período de $24 \mathrm{~h}$. Em seguida a solução foi centrifugada em centrífuga modelo MPW350 , e quantificou o corante remanescente por leitura direta em espectrofotômetro (Biochrom Libra S70), no comprimento de onda $665 \mathrm{~nm}$. Novamente, a eficiência de adsorção foi calculada utilizando a equação 1 .

\section{RESULTADOS E DISCUSSÃO}

\subsection{Caracterização da Sílica}

\subsubsection{Difração de Raios X (DRX)}

Através do estudo dos padrões de difração de raios $\mathrm{X}$ é possível inferir sobre o ordenamento estrutural dos materiais obtidos. Dessa forma, foram obtidos difratogramas das silicas calcinadas a $80^{\circ} \mathrm{C}, 105^{\circ} \mathrm{C}$ e $120^{\circ} \mathrm{C}$ em ângulos baixos e ângulos maiores que $10^{\circ}$. Os difratogramas das amostras de sílica (Figura 1) apresentaram três picos resolvidos que podem ser indexados com os planos (100), (110) e (200), associados com a simetria hexagonal 2-D p6mm, indicando a presença de um arranjo nanoporoso ordenado no material sintetizado (CAO, 2007). Nenhum pico foi encontrado, em ângulos superiores a $2 \theta=10^{\circ}$ (não mostrado), indicando a ausência de fases cristalinas na amostra e mostrando que a SBA-15 foi obtida como uma fase pura.

Figura 1 - Difratogramas de raios $X$ em ângulos baixo das amostras de sílica SBA-15.

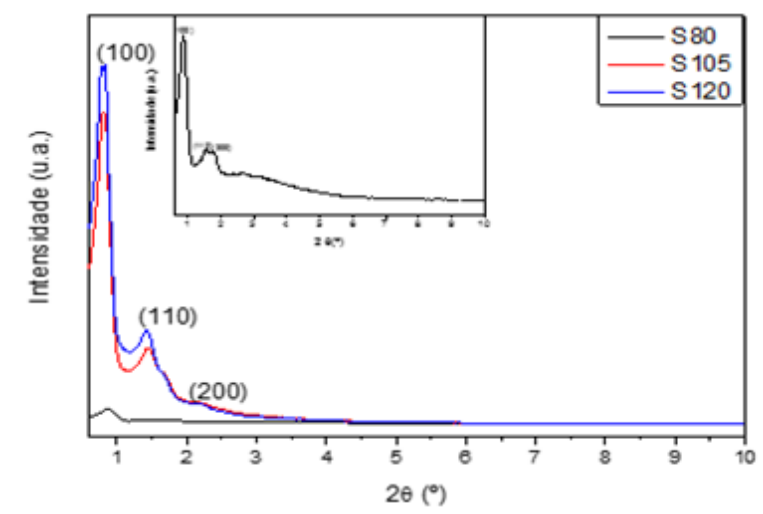

Fonte: Dados da pesquisa.

3.1.2 Medida de Porosidade e Área Superficial Específica 
Analisando os resultados apresentados na Tabela 1 , pode-se notar que a amostra sintetizada a $80^{\circ} \mathrm{C}$ foi a que apresentou maior área superficial devido a presença de microporos em sua estrutura. A amostra sintetizada a $120{ }^{\circ} \mathrm{C}$ apresentou um valor de área intermediário, maior diâmetro de poros e volume de mesoporos. Segundo Zhao et al. (1998) o aumento da temperatura de síntese favorece o aumento dos poros da sílica. Esse fato pode ser comprovado pelos resultados encontrados.

Tabela 1 - Propriedades texturais das sílicas mesoporosas SBA-15.

\begin{tabular}{ccccc}
\hline Amostra & $\begin{array}{c}\mathbf{S}_{\mathbf{g}} \\
\left(\mathbf{m}^{2} / \mathbf{g}\right)\end{array}$ & $\begin{array}{c}\mathbf{D}_{\mathbf{p}} \\
(\mathbf{n m})^{\mathbf{a}}\end{array}$ & $\begin{array}{c}\mathbf{V}_{\text {meso }} \\
\left(\mathbf{c m}^{3} / \mathbf{g}\right)\end{array}$ & $\begin{array}{c}\mathbf{V}_{\text {Micro }} \\
\left(\mathbf{c m}^{3} / \mathbf{g}\right)\end{array}$ \\
\hline $\mathbf{S 8 0}$ & 648 & 6,08 & 0,80 & 0,022 \\
$\mathbf{S 1 0 5}$ & 160 & 6,62 & 0,28 & - \\
$\mathbf{S 1 2 0}$ & 493 & 8,55 & 1,45 & - \\
\hline
\end{tabular}

Fonte: Dados da pesquisa.

*As siglas S80, S105 E S120 representam as sílicas sintetizadas a $80^{\circ} \mathrm{C}, 105^{\circ} \mathrm{C}$ e $120^{\circ} \mathrm{C}$, respectivamente. ${ }^{\mathrm{a}}$ Máximo da distribuição de tamanho de poro;

\subsection{Obtenção das Condições Ótimas de Adsorção}

Estão apresentados na Tabela 2 os resultados obtidos para os testes adsortivos do azul de metileno nas três concentrações utilizadas e nas três amostras de sílica SBA15.

Observa-se que independentemente da temperatura de síntese foi possível obter sílicas com alta eficiência de adsorção. Porém, a sílica sintetizada a $80^{\circ} \mathrm{C}$ foi a que apresentou maior eficiência nas três concentrações de azul de metileno utilizadas. Tal resultado pode ser explicado a partir da análise da Tabela 1. Como a sílica sintetizada a $80^{\circ} \mathrm{C}$ obteve uma maior área superficial, provavelmente possuiu mais sítios de ligação para o azul de metileno, favorecendo uma maior adsorção. A combinação que gerou uma maior eficiência foi a sílica sintetizada a $80^{\circ} \mathrm{C}$ com concentração de corante de 100 mg. $\mathrm{L}^{-1}$, sugerindo ser essa combinação de amostra e concentração a ideal à ser utilizada na etapa posterior. Mesmo em concentrações elevadas de corante a eficiência foi sempre superior a $90 \%$.

Tabela 2 - Resultados da eficiência de adsorção da sílica SBA-15 nas três temperaturas de síntese e nas três concentrações de corante utilizadas.

\begin{tabular}{ccc}
\hline Amostra & $\begin{array}{c}\text { Concentração do azul } \\
\text { de metileno }\left(\text { mg.. }^{\mathbf{1}} \text { ) }\right.\end{array}$ & $\begin{array}{c}\text { Eficiência } \\
(\%)\end{array}$ \\
\hline S80 & 100 & 98,99 \\
S80 & 200 & 98,13 \\
S80 & 300 & 96,83 \\
S105 & 100 & 97,63 \\
S105 & 200 & 97,15 \\
S105 & 300 & 96,04 \\
S120 & 100 & 96,08 \\
S120 & 200 & 96,21 \\
S120 & 300 & 94,16 \\
\hline
\end{tabular}

Fonte: Dados da pesquisa.

*As siglas S80, S105 E S120 representam as sílicas sintetizadas a $80^{\circ} \mathrm{C}, 105^{\circ} \mathrm{C}$ e $120^{\circ} \mathrm{C}$, respectivamente.

Na Tabela 3 são apresentados os resultados médios do estudo de $\mathrm{pH}$, utilizando a sílica sintetizada a $80^{\circ} \mathrm{C}$ e concentração da solução de azul de metileno de $100 \mathrm{mg} \cdot \mathrm{L}^{-1}$.

Tabela 3 - Resultados da eficiência de adsorção da sílica SBA-15 na temperatura de síntese de $80^{\circ} \mathrm{C}$ com variação de $\mathrm{pH}$.

\begin{tabular}{ccc}
\hline Amostra & pH & Eficiência (\%) \\
\hline S80 & 3,00 & 96,84 \\
S80 & 7,00 & 98,99 \\
S80 & 9,00 & 99,74 \\
\hline
\end{tabular}

Fonte: Dados da pesquisa.

*As siglas S80 representa a sílica sintetizada a $80^{\circ} \mathrm{C}$.

Foi obtida uma alta eficiência de adsorção do azul de metileno nos três valores de $\mathrm{pH}$ estudados, sugerindo que a sílica possui 
superficiais negativas, pelo fato de o azul de metileno ser uma espécie catiônica. $\mathrm{O}$ percentual de remoção mais baixo em meio ácido pode estar associado com a protonação do corante e à presença de excesso de íons $\mathrm{H}^{+}$ competindo com os cátions do corante pelos sítios ativos (FOO e HAMEED, 2012). Nos valores de $\mathrm{pH}$ 7,00 e 9,00 o azul de metileno encontra-se desprotonado $\left(\mathrm{pK}_{\mathrm{a}}=5,60\right)$, mas ainda assim apresenta cargas positivas por se tratar de uma espécie catiônica. Observa-se que a medida que se aumentou o $\mathrm{pH}$ da solução de azul de metileno a eficiência de adsorção também foi aumentada.

\section{CONCLUSÃO}

A partir do estudo de caracterização foi possível verificar que a sílica sintetizada a $80^{\circ} \mathrm{C}$ apresentou uma maior área superficial, menor diâmetro de poros, além da presença de microporos em sua estrutura. Realizou-se os testes iniciais de adsorção e observou-se que a concentração a ser utilizada para o azul de metileno foi de $100 \mathrm{ppm}$, utilizando a sílica calcinada a $80^{\circ} \mathrm{C}$, pois foi com essa combinação que os maiores valores de eficiência foram alcançados. Em seguida, foi realizado um estudo de $\mathrm{pH}$ e foi possível verificar que a solução de azul de metileno no pH 9,0 foi o obteve a maior adsorção.

\section{NOMENCLATURA}

S80 - sílicas sintetizadas a $80^{\circ} \mathrm{C}$;

S105 - sílicas sintetizadas a $105^{\circ} \mathrm{C}$;

$\mathrm{S} 120$ - sílicas sintetizadas a $120^{\circ} \mathrm{C}$;

efic - eficiência da adsorção (\%);

$\mathrm{V}$ (mL) -volume da solução;

$\mathrm{C}_{\text {in }}\left(\mathrm{mg} . \mathrm{L}^{-1}\right)$ - concentração inicial da solução;

C (mg. $\left.L^{-1}\right)$ - concentração da solução no equilíbrio;

$\mathrm{S}_{\mathrm{g}}\left(\mathrm{m}^{2} / \mathrm{g}\right)$ - área superficial;

$\mathrm{D}_{\mathrm{p}}(\mathrm{nm})^{\mathrm{a}}$ - diâmetros dos poros;

$\mathrm{V}_{\text {meso }}\left(\mathrm{cm}^{3} / \mathrm{g}\right)$ - volume de mesoporos;

$\mathrm{V}_{\text {Micro }}\left(\mathrm{cm}^{3} / \mathrm{g}\right)$ - volume de microporos.

\section{REFERÊNCIAS}

BARRET, E. P.; JOYNER, L. G.; HALENDA, P. P. The determination of pore volume and area distributions in porous substances. I. Computations from nitrogen isotherms, Journal of The American Chemical Society, v. 73, p. 373-380, 1951.

BRUNAUER, S.; EMMET, P. H.; TELLER, E. Adsorption of Gases in multimolecular layers, Journal of The American Chemical Society, v. 60, p. 309-319, 1938.

CAO, Y.; CAO, J.; ZHENG, M.; LIU, J.; JI, G. Synthesis, characterization, and electrochemical properties of ordered mesoporous carbons containing nickel oxide nanoparticles using sucrose and nickel acetate in a silica template, Journal of Solid State Chemistry, v. 180, p. 792-798, 2007.

FOO, K. Y.; HAMEED, B. H. Adsorption characteristics of industrial solid waste derived activated carbon prepared by microwave heating for methylene blue. Fuel Processing Technology, v. 99, p. 103-109, 2012.

LIPPENS, B. C.; LINSEN, B. G.; BÔER, J. H. Studies on pore systems in catalysts I. The adsorption of nitrogen; apparatus and calculation, Journal of Catalysis, v. 3, p. 3237, 1964.

MASEL, R. I. Principles of Adsorption and reaction on solid surfaces, John Wiley e Sons Inc., p. 112, 1996.

YANG, H.; COOMBS, N.; OZIN, G.A. Morphogenesis oh shapes and surfasse patterns in mesoporoussílica. Nature, v. 386, p. 692-695, 1997. 
ZHAO, D.; FENG, J.; HUO, Q.; MELOSH, N.; FREDRICKSON, G.H.; CHMELKA, B.F.; STUCKY, G.D. Triblock copolymer syntheses of mesoporous sílica with periodic 50 to 300 angstrom pores. Science, v. 279, p. 548-552, 1998. 